\title{
Structure and genetic stability of mitochondrial genomes vary among yeasts of the genus Saccharomyces
}

\author{
Jure Piškur, ${ }^{1,2}$ Sonja Smole, ${ }^{2}+$ Casper Groth, ${ }^{1,2}$ Randi F. Petersen ${ }^{1,2}$ \\ and Mogens B. Pedersen ${ }^{3}$
}

Author for correspondence: Jure Piškur. Tel: +4545 252518. Fax: +4545932809.

e-mail: jp@im.dtu.dk

1 Department of

Microbiology, Technical University of Denmark, DTU-301, DK-2800 Lyngby, Denmark

2 Department of Genetics, Institute of Molecular Biology, University of Copenhagen, Denmark

3 Carlsberg Research Laboratory, Denmark
Several yeast species/isolates belonging to the genus Saccharomyces were examined for the organization of their mtDNAs and ability to generate petite mutants. A general characteristic for all of the mtDNAs tested was that they were very A+T-rich. However, restriction patterns and inducibility of petite mutations revealed a great diversity in the organization and genetic behaviour of mtDNAs. One group of yeasts, Saccharomyces sensu stricto, contains mtDNA ranging in size from 64 to $85 \mathrm{~kb}$. mtDNAs from these yeasts contain a high number of restriction sites that are recognized by the enzymes Haelll and Mspl, which cut specifically in $\mathrm{G}+\mathrm{C}$ clusters. There are three to nine orilrep sequences per genome. These yeasts spontaneously generate respiration deficient mutants. Ethidium bromide (Et-Br), at low concentrations, induces a majority of cells to give rise to petites. A second group of yeasts, Saccharomyces sensu lato, contains smaller mtDNAs, ranging in size from 23 to $48 \mathrm{~kb}$, and probably only a few intergenic $\mathrm{G}+\mathrm{C}$ clusters and no orilrep sequences. These yeasts also generate petite clones spontaneously, but Et-Br, even when present at high concentrations, does not substantially increase the frequency of petites. In most petite clones from these yeasts only a small fragment of the wild-type molecule is retained and apparently multiplied. A third group, represented by Saccharomyces kluyveri, does not give rise to petite mutants either spontaneously or after induction.

Keywords: yeast, mitochondrial genome, petite mutation, intergenic sequences, taxonomy

\section{INTRODUCTION}

A remarkable aspect of mitochondrial genomes of all organisms is that they contain a very similar set of genes. On the other hand, mtDNA molecules among diverse species are highly variable in size and organization $(5,36)$. The yeast Saccharomyces cerevisiae has played the central role in studies of mtDNA heredity (for reviews, see 9,28 ). This is due mainly to the property that this yeast is a facultative anaerobe; i.e. it can survive without active mitochondria. The average cell of $S$. cerevisiae contains as many as $50 \mathrm{mtDNA}$ molecules, but the number varies

†Present address: Department of Food Science and Technology, Biotechnical Faculty, University of Ljubljana, 1000 Ljubljana, Slovenia. with the genotype and physiological conditions (for a review, see 14). mtDNA of $S$. cerevisiae is characterized by a very low $\mathrm{G}+\mathrm{C}$ content $(18 \mathrm{~mol} \%)$, and clustering of $\mathrm{G}+\mathrm{C}$ - and $\mathrm{A}+\mathrm{T}$-rich regions (37). Most methods of preparation yielded exclusively linear molecules, but mapping and sequencing of the genome showed that the genetic map of mtDNA is circular (9). The 'long' version of the genome, containing additional introns and intergenic sequences, is $85 \mathrm{~kb}$ in size and the 'short' version is $74 \mathrm{~kb}(37)$. Genes represent less than one-fifth of the mtDNA molecule, the rest consists of introns and intergenic sequences. The intergenic regions represent about two-thirds of mtDNA and consist of repetitive $\mathrm{A}+\mathrm{T}$-rich spacers and short sequences with $\mathrm{a}+\mathrm{C}$ content greater than $50 \mathrm{~mol} \%$, so-called G $+\mathrm{C}$ clusters. Approximately 200 $\mathrm{A}+\mathrm{T}$ spacers with a mean size of $190 \mathrm{bp}$ represent 
almost half of the genome, and they are usually separated from each other by over $200 \mathrm{G}+\mathrm{C}$ clusters (38). A large fraction of $\mathrm{G}+\mathrm{C}$ clusters contains recognition sites for restriction enzymes splitting target sequences which contain only $\mathrm{G}$ and $\mathrm{C}$ residues, i.e. HaeIII and HpaII/MspI (38). A special class of $\mathrm{G}+\mathrm{C}$ clusters are ori/rep sequences which are about $300 \mathrm{bp}$ long and are present in eight copies scattered around the genome $(4,40) . \mathrm{G}+\mathrm{C}$ clusters can be grouped into eight families which presumably originated from a proto-G $+\mathrm{C}$ cluster (38). Therefore, mtDNA contains a variety of short duplications which potentially can be involved in intramolecular recombination.

S. cerevisiae spontaneously produces mutants, petites, which are deficient in the ability to respire aerobically. The spontaneous frequency is about $1 \%$, but upon induction with chemical mutagens, e.g. ethidium bromide (Et-Br), all cells may give rise to petites (for reviews, see $2,13,14)$. The petite phenotype is correlated with gross alterations, extensive deletions or loss of mtDNA (2). These deletions may be accompanied by reiteration of the remaining wild-type segments, so that some petite mutants contain the same amount of mtDNA as wild-type strains $(2,33)$. The sequences involved in excision of petite mtDNA are short direct repeats located in the $\mathrm{A}+\mathrm{T}$ spacers and $\mathrm{G}+\mathrm{C}$ clusters $(8,39)$. Therefore, the intergenic sequences are directly involved in destabilization of the mitochondrial genome. Once a petite mtDNA molecule is generated, it may gain exclusive representation in a daughter cell.

The majority of spontaneous petite mtDNA molecules exhibit a high transmission capacity in genetic crosses. In crosses with wild-type strains, such petites give a substantial proportion of petite progeny (for a review, see 14). A heteroplasmic cell, containing two different types of mtDNA molecules, eventually produces offspring that are homoplasmic and contain only one type of mtDNA. Homoplasmic daughter cells can be found after just four generations, suggesting that transmission is an active process (14). Apparently, $\mathrm{G}+\mathrm{C}$ clusters, especially the ori/rep sequences, represent active elements which facilitate transmission of a particular mtDNA molecule $(4,25,40)$. However, the overall structure of mtDNA also seems to be important in transmission $(11,26,31)$.

Previously, it was not known whether the observations on the structure and genetic stability of mtDNA of $S$. cerevisiae could be extrapolated to other closely related yeasts. The genus Saccharomyces can be divided into two major groups: sensu stricto (including $S$. cerevisiae) and sensu lato $(1,20)$. In the present study the type strain and some additional isolates of all currently recognized species of the genus Saccharomyces were examined with regard to some characteristics of their mtDNA and their ability to generate petites. Apparently, at least three categories with differing organization and genetic stability of mtDNA exist in this genus. One has mtDNA larger than $64 \mathrm{~kb}$, contains $\mathrm{G}+\mathrm{C}$ clusters and is highly inducible for petite generation. The second category contains mtDNA molecules with fewer than $50 \mathrm{~kb}$ and is less inducible for petite generation. The third category is represented by a single petite-negative member.

\section{METHODS}

Yeast strains. The following Saccharomyces yeast type isolates were studied for their mtDNA molecules: Saccharomyces bayanus (CBS 380 ${ }^{\mathrm{T}}$ ), Saccharomyces carlsbergensis (CBS 1513 $)$, Saccharomyces castellii (NRRL Y-12630 $)$, S. cerevisiae (NRRL Y-12632 ${ }^{\mathrm{T}}$ ), Saccharomyces dairensis (NRRL Y-12639 $)$, Saccharomyces douglasii (CBS 2908), Saccharomyces exiguus (NRRL Y-12640 $)$, Saccharomyces kluyveri (NRRL Y-12651 ${ }^{\mathrm{T}}$ ), Saccharomyces monacensis (CBS 1503), Saccharomyces paradoxus (NRRL $\mathrm{Y}-17217^{\mathrm{T}}$ ), Saccharomyces pastorianus (CBS 1538), Saccharomyces servazzii (NRRL Y-12661 ${ }^{\mathrm{T}}$ ), Saccharomyces unisporus (NRRL Y-1556 $)$, Saccharomyces uvarum (CBS 395). ' $T$ ' indicates a type strain, and represents a separate species, but some isolates, as will be shown below, apparently belong to the same species. The strains carrying the CBS designation were obtained from the Centraal Bureau voor Schimmelcultures, Delft, The Netherlands. The NRRL Ystrains originate from the National Center for Agricultural Utilization Research, Peoria, IL, USA. In addition, a laboratory strain of $S$. cerevisiae, T3/3 (10), was used as a standard in several experiments.

mtDNA isolation. For isolation of DNA, a culture was pregrown in YPD medium ( $2 \%$ glucose, $0.5 \%$ yeast extract, $1.0 \%$ peptone) and then grown overnight in GlyYP (2\% glycerol, $0.5 \%$ yeast extract, $1.0 \%$ peptone) at $25^{\circ} \mathrm{C}$. Spheroplasts were prepared using zymolyase and lysed with SDS. mtDNA was separated from the other DNA using a bisbenzimide- $\mathrm{CsCl}$ gradient (27). At least two bands appeared in the gradient and they were tested for crosshybridization with the $S$. cerevisiae mitochondrial probes. When the bands were poorly resolved, the upper fraction was isolated and re-subjected to centrifugation in a $\mathrm{CsCl}$ gradient.

DNA techniques. mtDNA was digested with various restriction enzymes, and DNA fragments were separated by electrophoresis in $0.8-1.2 \%$ agarose gels. Lambda DNA digested with various restriction enzymes was used as the size marker. The size of mtDNA was calculated from several independent digestions using at least five different restriction enzymes. DNA was transferred from gels to Hybond-N+ (Amersham) membranes under alkaline conditions. Hybridization was performed at $55^{\circ} \mathrm{C}$ with radioactive probes prepared by the random priming method. The following probes were used: plasmids carrying $S$. cerevisiae mitochondrial $\operatorname{LrRNA}$ or $\operatorname{Sr} R N A$ genes (obtained from $\mathrm{H}$. Fukuhara, Orsay, France) and petite mtDNA carrying the $S$. cerevisiae oril sequence (3). These mitochondrial probes helped to distinguish mtDNA from other DNA bands present in the $\mathrm{CsCl}$ gradient.

$\mathbf{G}+\mathbf{C}$ content. The $\mathrm{G}+\mathrm{C}$ content of mtDNA was determined using $\mathrm{CsCl}$ buoyant density centrifugation. Pure mtDNA, isolated from a $\mathrm{CsCl}$ gradient was mixed with purified nuclear DNA from $S$. cerevisiae and run again in the ultracentrifuge. The $\mathrm{G}+\mathrm{C}$ contents of non-S. cerevisiae mtDNAs were determined using $S$. cerevisiae nuclear $(40$ $\mathrm{mol} \% \mathrm{G}+\mathrm{C})$ and mtDNA $(18 \mathrm{~mol} \% \mathrm{G}+\mathrm{C})$ as standards 
(37). It was assumed that the density of mtDNA in a band can be determined from a linear function of its distance from the bands of both standards (21). An error of this method has been estimated to be $\pm 0.5 \mathrm{~mol} \%$ of the $\mathrm{G}+\mathrm{C}$ content.

$\mathbf{G}+\mathbf{C}$ clusters. If the sequence of nucleotides is completely random the number of sites for any particular restriction enzyme can be predicted. In the case of ' $\mathrm{G}+\mathrm{C}$-cutters', i.e., HaeIII and MspI/HpaII, which contain four bases in the target site, the following equation can be used:

Number of sites $=$ number of base pairs

$$
\times(\mathrm{G}+\mathrm{C} \text { content } / 2)^{4} \text {. }
$$

However, in $S$. cerevisiae a wide discrepancy between the observed and the predicted numbers of sites of HaeIII and HpaII (MspI) exists, because of clustering of $\mathrm{G}$ and $\mathrm{C}$ residues.

Spontaneous petite mutations. The spontaneous frequency of petites was determined by plating yeast cultures on the medium containing a limiting amount of a fermentable carbon source. Yeasts pre-grown in YPD were diluted and plated on GGlyYP ( $2 \%$ glycerol, $0.1 \%$ glucose, $0.5 \%$ yeast extract, $1 \%$ peptone and $1.5 \%$ agar). After a few days of growth, the frequency of small colonies, putative petite mutants, was determined. These colonies were examined for growth on GlyYP and tested for respiratory potential using the tetrazolium method (22). mtDNA was isolated from some of the non-respiratory colonies and examined with several restriction enzymes.

Induced petite mutations. The induction of petites by treatment with Et-Br was studied. Various amounts of Et-Br were added to exponentially growing yeast cells in YPD, and growth was continued for an additional $8 \mathrm{~h}$. During this period cells should normally divide three or four times. If a petite mutation was induced at the beginnimg of the treatment, it could have segregated into a homoplasmic clone by the end of the treatment. Heteroplasmic cells containing a mix of wild-type and petite mtDNA at the time of plating, would generate colonies which contained respiration-competent and -deficient cells. Such colonies would in part respire, and they would be scored as respiration-competent. Following the Et-Br treatment the cells were washed and plated on the GGlyYP medium. After several days the frequency of petites was determined.

\section{RESULTS AND DISCUSSION}

\section{mtDNA molecules vary in size}

When total DNA from different yeast isolates was separated on a $\mathrm{CsCl}$ gradient, at least two distinct bands appeared. The positions of these bands in the gradients varied slightly and, in addition, the distance between them was variable. The pattern with two bands resembled the pattern known in $S$. cerevisiae where the upper band represents mtDNA and the lower one nuclear DNA (for a review, see 9). The upper band from various yeast isolates always gave a positive reaction when hybridized to the $S$. cerevisiae mitochondrial gene probes. The lower band, which was stronger in intensity, did not hybridize to the $S$. cerevisiae mitochondrial probes, and it presumably represented nuclear DNA (data not shown). Some-

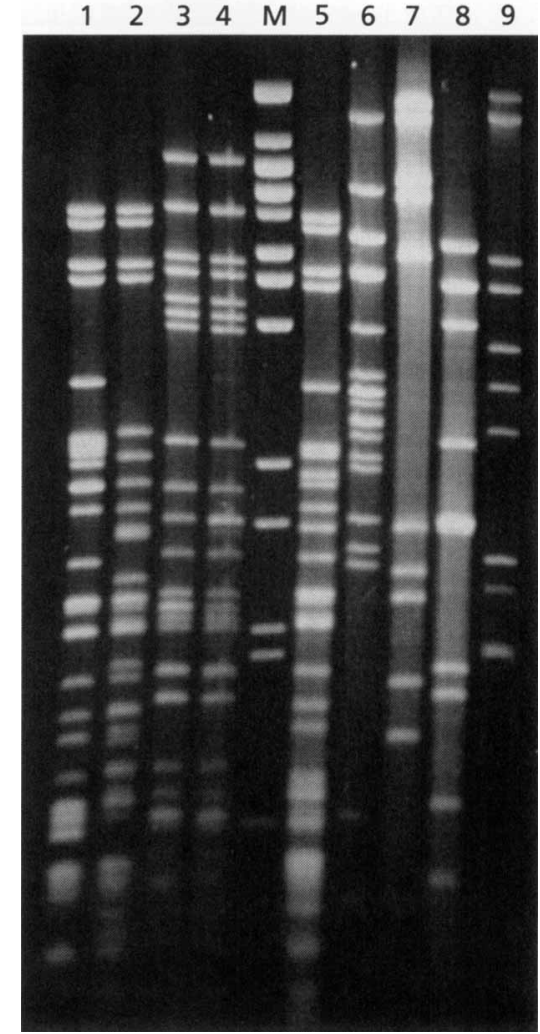

Fig. 1. mtDNA isolated from $S$. monacensis (1), S. carlsbergensis (2), S. uvarum (3), S. bayanus (4), S. pastorianus (5), S. paradoxus (6), S. dairensis (7), S. castellii (8) and S. kluyveri (9) was digested with Haelll and the restriction fragments separated on a $1 \%$ agarose gel. Lambda DNA cut with BstEll was used as a marker, $\mathrm{M}$.

times, with DNA from $S$. exiguus, $S$. servazzii and $S$. unisporus, three DNA bands appeared in $\mathrm{CsCl}$ gradients. The two upper bands were located close to each other and they both hybridized to the $S$. cerevisiae mtDNA probes. The basis for the existence of double mtDNA bands is not understood; perhaps it reflects the presence of two or more different physical forms of the mtDNA molecule. In further studies to determine the size, restriction pattern and $\mathrm{G}+\mathrm{C}$ content, only the upper of these two mtDNA bands was investigated.

When various mtDNAs were digested by different restriction enzymes, almost all of the tested isolates provided a specific restriction pattern. If the restriction enzyme cut several times, species/isolate-specific restriction fingerprints resulted (Figs 1 and 2). However, in the cases of (i) $S$. bayanus and $S$. uvarum, and (ii) $S$. carlsbergensis, S. monacensis and S. pastorianus, respectively, the patterns were identical or quite similar (Fig. 1 and 2). Obviously, mtDNA molecules which showed similarities in restriction patterns are related, and the present results could indicate that the yeasts yielding similar patterns belong to the same or closely related species (Figs 1 and 2). These results are in 


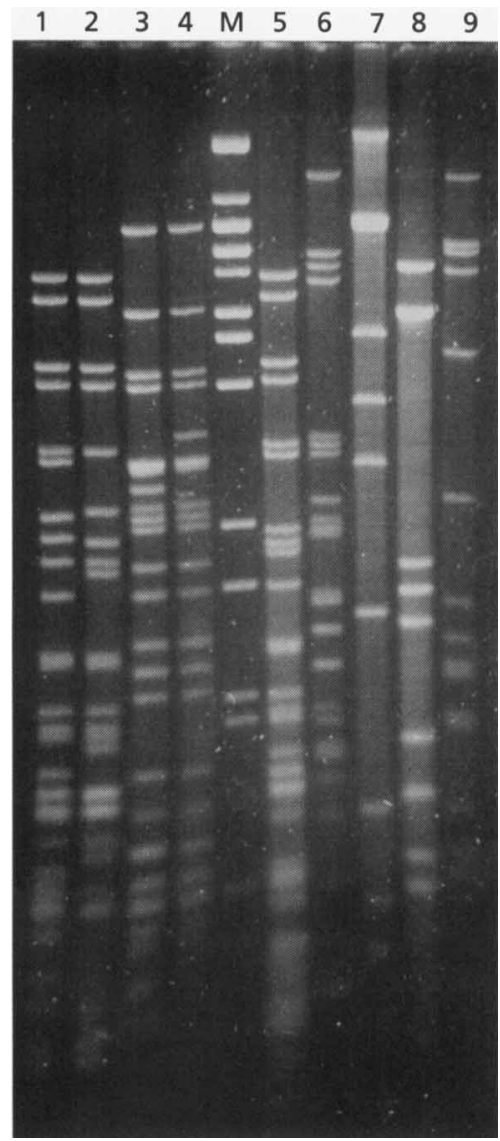

Fig. 2. mtDNA isolated from various yeasts (see legend to Fig. 1) and cut with $\mathrm{Mspl}$; otherwise the same as for Fig. 1.

agreement with the previous observations on these isolates $(1,20)$.

The most striking observation, when the restriction pattern from various yeast isolates was compared, was that the number of restriction fragments varied substantially (Figs 1 and 2). Thus, the size of mtDNAs among these yeasts is different. The total size of mtDNA was determined by at least five restriction enzymes used in various combinations (data not shown). A group of yeasts belonging to the sensu stricto group showed the size of mtDNA above $64 \mathrm{~kb}$ (Table 1). The observed sizes in $S$. bayanus, $S$. carlsbergensis, $S$. monacensis, $S$. pastorianus, $S$. paradoxus and $S$. uvarum are very similar to the sizes previously reported for $S$. cerevisiae and $S$. douglasii $(34,37)$. On the other hand, isolates belonging to the sensu lato group, had smaller mtDNA molecules below $50 \mathrm{~kb}$ (Table 1 ). In the case of $S$. exiguus and $S$. castellii the sizes found were only $23 \mathrm{~kb}$ and $26 \mathrm{~kb}$, respectively. Thus, the proportion of DNA in intergenic sequences in these two species must be drastically lower than that in the sensu stricto group. $S$. servazzii and $S$. unisporus have the same size mtDNA, namely $29 \mathrm{~kb}$, which is only slightly larger than that of $S$. castellii and $S$. exiguus. Even if $S$. servazzii and $S$. unisporus are closely related (20), their restriction patterns are substantially different (data not shown). S. dairensis and S. kluyveri contain larger mtDNA molecules of 48 and $49 \mathrm{~kb}$, respectively, but their restriction patterns were completely different (Table 1, Figs 1 and 2). In conclusion, a great heterogeneity with regard to size and organization of mtDNA exists within the genus $\mathrm{Sac}$ charomyces. The mtDNA molecules were examined further to determine other physical characteristics.

\section{$\mathbf{G}+\mathbf{C}$ content, $\mathbf{G}+\mathbf{C}$ clusters and ori/rep}

The $\mathrm{G}+\mathrm{C} / \mathrm{A}+\mathrm{T}$ ratios of mtDNA molecules were determined using $\mathrm{CsCl}$ gradients and $S$. cerevisiae mtDNA and nuclear DNA as standards. The $\mathrm{G}+\mathrm{C}$ content of yeasts belonging to the sensu stricto group is $18 \mathrm{~mol} \%$ or less, while sensu lato yeasts have a slightly higher $\mathrm{G}+\mathrm{C}$ content, ranging from 19 to $24.5 \mathrm{~mol} \%$ (Table 2). As mentioned above, in a few cases, $S$. exiguus, $S$. servazzii and $S$. unisporus, two bands containing mtDNA sometimes appeared in the $\mathrm{CsCl}$ gradient. In this case only the $\mathrm{G}+\mathrm{C}$ content of the upper band was determined and is shown in Table 2 .

There is a clear tendency in all members of the genus Saccharomyces for mtDNA to exhibit a very low $\mathrm{G}+\mathrm{C}$ content (Table 2). Some other yeasts, for example Pachytichospora transvaalensis, have a much higher $\mathrm{G}+\mathrm{C}$ content (6). Thus, a general mechanism which ensures a very low $\mathrm{G}+\mathrm{C}$ content in the mtDNA molecule must exist in the genus Saacharomyces.

$\mathrm{G}$ and $\mathrm{C}$ residues could be dispersed randomly in the genome or clustered as is the case with the $S$. cerevisiae mtDNA molecule. In $S$. cerevisiae a majority of these $G+C$ clusters contain motifs which are recognized by $\mathrm{G}+\mathrm{C}$-cutting restriction enzymes. The isolated mtDNA molecules were examined for the presence of HaeIII and MspI restriction sites (Figs 1 and 2). In general, the patterns obtained could be divided into two groups: sensu stricto yeasts gave 25 or more restriction fragments, and sensu lato yeasts below 20 (Figs 1 and 2). On the basis of the sizes and $\mathrm{G}+\mathrm{C}$ contents of the various mtDNAs, it was possible to calculate the number of restriction sites for molecules with a random distribution of $\mathrm{G}$ and $\mathrm{C}$ residues (Table 2). It should be noted that for mtDNAs of the sensu stricto group it was difficult to determine the precise number of fragments, because some were too small or they overlapped with others on the gels. In the group of sensu stricto yeasts the predicted number of fragments is three or four (Table 2), and this is about one order of magnitude lower than the observed number of sites (Figs 1 and 2). The numbers of ori/rep sequences occurring in these yeasts were also estimated (Table 1). S. cerevisiae has eight ori/rep sequences which exhibit some sequence polymorphism (12). When $S$. cerevisiae mtDNA was digested with different restriction enzymes, up to eight different restriction fragments in some digests gave a positive signal with 
Table 1. The size, number of restriction sites recognized by $\mathrm{G}+\mathrm{C}$-cutting restriction enzymes, Haell and Mspl, and number of orilrep sequences in the mitochondrial DNA molecule of yeasts belonging to the genus Saccharomyces

\begin{tabular}{|lcrcr|}
\hline Yeast & $\begin{array}{c}\text { Size* of } \\
\text { mtDNA (kb) }\end{array}$ & \multicolumn{3}{c|}{ Number of sites } \\
\cline { 3 - 5 } & & MspI & HaeIII & ori/rep \\
\hline Saccharomyces sensu stricto & & & & \\
S. bayanus & $64 \cdot 3$ & $>35$ & $>25$ & 4 \\
S. carlsbergensis & $66 \cdot 6$ & $>40$ & $>40$ & 4 \\
S. cerevisiae* $_{\text {S. douglasii* }}$ & $78-85$ & $>45$ & $>45$ & 8 \\
S. monacensis & 73 & $>35$ & $>20$ & 6 \\
S. paradoxus & $66 \cdot 6$ & $>40$ & $>40$ & 5 \\
S. pastorianus & $67 \cdot 1$ & $>25$ & $>25$ & 7 \\
S. uvarum & $66 \cdot 6$ & $>45$ & $>25$ & 5 \\
Saccharomyces sensu lato & $64 \cdot 3$ & $>40$ & $>30$ & 4 \\
S. castellii & & & & \\
S. dairensis & 26 & 10 & 10 & ND \\
S. exiguus* & 48 & 10 & 10 & ND \\
S. kluyveri & 23 & 3 & 3 & ND \\
S. servazzii & 49 & 17 & 12 & ND \\
S. unisporus & 29 & 15 & 10 & ND \\
\hline
\end{tabular}

ND, Not detected.

*The sizes of mtDNA from $S$. cerevisiae, $S$. douglasii and $S$. exiguus have been determined previously and can be found elsewhere $(7,34,37)$.

Table 2. $\mathrm{G}+\mathrm{C}$ contents of various mtDNA molecules, and the predicted number of restriction sites recognized by the $\mathrm{G}+\mathrm{C}$-cutters, Haell and Mspl, if the distribution of $\mathrm{G}$ and $\mathrm{C}$ residues is random

\begin{tabular}{|lcc|}
\hline Yeast & $\begin{array}{c}\text { G }+ \text { C content of mtDNA } \\
\text { (mol \%) }\end{array}$ & $\begin{array}{c}\text { Predicted no. of HaeIII or } \\
\text { Mspl sites }\end{array}$ \\
\hline Saccharomyces sensu stricto & & \\
S. bayanus & 18 & 4 \\
S. carlsbergensis & 18 & 4 \\
S. cerevisiae & 18 & 4 \\
S. monacensis & 18 & 4 \\
S. paradoxus & 16 & 3 \\
Saccharomyces sensu lato & & \\
S. castellii & 22 & 4 \\
S. dairensis & 19 & 4 \\
S. exiguus & 22 & 3 \\
S. kluyveri & 22 & 7 \\
S. servazzii & $24 \cdot 5$ & 7 \\
S. unisporus & 23 & 5 \\
\hline
\end{tabular}

the ori/rep probe. The approximate number of the ori/rep sequences was estimated in a similar way in other species. The maximum number of fragments giving a positive signal upon examining several digests was then taken as the number of sequences per genome. The number varied from four to eight among different sensu stricto species (Table 1). Isolates which seem to be more closely related on the basis of their nuclear rRNA sequences (20), also showed similar numbers of ori/rep sequences. For example, $S$. bayanus and $S$. uvarum both contain four ori/rep sequences, and $S$. douglasii and $S$. paradoxus contain six or seven ori/rep sequences, respectively. In addition, $S$. cerevisiae and $S$. paradoxus, which are very closely related (20), 
Table 3. Frequency of petites (\%) in various yeasts upon induction with ethidium bromide (Et-Br)

Yeasts were grown in the presence of various concentrations of Et-Br for $8 \mathrm{~h}$ and then plated on selective medium.

\begin{tabular}{|llrrrrr|}
\hline Yeast & \multicolumn{7}{c|}{ Et-Br concn $\left(\mathbf{m g ~ I}^{\mathbf{- 1}}\right)$} \\
\cline { 2 - 7 } & $\mathbf{0}$ & $\mathbf{5}$ & $\mathbf{1 0}$ & $\mathbf{1 5}$ & $\mathbf{2 5}$ & $\mathbf{5 0}$ \\
\hline Saccharomycces sensu stricto & & & & & & \\
S. bayanus & $4 \cdot 5$ & 10 & - & 96 & 100 & 100 \\
S. cerevisiae & $0 \cdot 14$ & 2 & - & 94 & 100 & 100 \\
S. monacensis & $3 \cdot 2$ & 4 & 5 & 42 & 81 & 97 \\
S. pastorianus & $4 \cdot 8$ & - & 35 & 97 & 98 & 100 \\
S. uvarum & $6 \cdot 7$ & 10 & - & 96 & 100 & 100 \\
Saccharomycces sensu lato & & & & & & \\
S. castellii & $5 \cdot 7$ & 6 & - & 6 & 18 & 24 \\
S. dairensis & 1.57 & 2 & - & 4 & 11 & 13 \\
S. exiguus & $1 \cdot 36$ & 1 & 4 & 4 & 6 & 9 \\
S. kluyveri & 0 & 0 & 0 & 0 & 0 & 0 \\
S. servazzii & $0 \cdot 13$ & 1 & 1 & 2 & 8 & 8 \\
\hline
\end{tabular}

resemble each other in the number of ori/rep sequences more than they resemble $S$. bayanus (Table 2).

In sensu lato yeasts the genome organization, so far as $\mathrm{G}+\mathrm{C}$ clusters are concerned, seems to be different from sensu stricto yeasts. While the genome size was smaller than that of the sensu stricto group, a similar number of HaeIII or $M s p I$ sites was predicted for sensu lato yeasts, because of a slightly higher $\mathrm{G}+\mathrm{C}$ content (Table 2). In contrast to the sensu stricto group, the discrepancy between the predicted and observed HaeIII and MspI sites is substantially smaller than in the sensu lato group. The obtained number of restriction fragments is only one to two and a half times higher than the number predicted on the basis of randomness. Thus, the distribution of $G$ and $C$ residues is likely to be more random in yeasts of the sensu lato group than of the sensu stricto group. Furthermore, in contrast to yeasts of the sensu stricto group, the sensu lato yeasts did not yield any signal with the ori/rep probe (Table 1). Therefore, two classes of mtDNA exist among yeasts belonging to the genus Saccharomyces. The sensu stricto group has a larger mtDNA molecule with abundant $\mathrm{G}+\mathrm{C}$ clusters recognized by HaeIII and $M s p \mathrm{I}$, and ori/rep sequences, while mtDNA of the sensu lato group is smaller, lacks ori/rep sequences, and apparently does not contain an extensive number of $\mathrm{G}+\mathrm{C}$ clusters with HaeIII and MspI sites.

In $S$. cerevisiae, $\mathrm{G}+\mathrm{C}$ clusters are involved in genetic instability of mtDNA through the promotion of the physical generation of petite mtDNA molecules and their enhanced transmission (28). Therefore, the two groups of yeasts, with different organizations of their mtDNA, were compared for their ability to generate petite mtDNA molecules.

\section{Genetic stability of mtDNA}

When stationary cultures of different yeast isolates were plated onto medium with a limited concentration of glucose (GGlyYP) in all cases except one large and small colonies appeared. These small colonies could not grow on glycerol medium, but could grow on the glucose medium. The smaller colonies, when transferred to plates containing tetrazolium remained colourless, indicative of their inability to respire aerobically. The frequency of the respiration deficient colonies varied from 0 in $S$. kluyveri to $6.7 \%$ in $S$. uvarum (Table 3 ). The mtDNA of several mutants was then examined. In some of the petite mutants mtDNA was not detected at all, either by analysis of total DNA with a molecular probe or by running a $\mathrm{CsCl}$ gradient (data not shown). Apparently, these mutants were $r h o^{\circ}$. However, a majority of respiratory-deficient colonies still yielded distinctive mtDNA bands in $\mathrm{CsCl}$ gradients, and these bands varied in intensity and position (data not shown). A few mtDNA molecules from the non-respiring strains of various species were subjected to restriction analysis. When restriction patterns were compared with those of the respective wild-type strains of the corresponding species, most of the fragments characteristic for the wild-type mtDNA molecules were missing from the patterns of the mutants (data not shown). Usually, only a very limited number of restriction sites was retained in petite mtDNA. The mutant analysed in Fig. 3 retained only three restriction sites. When this mtDNA was cut with any of these three restriction enzymes, a fragment of about $1 \mathrm{~kb}$ was obtained. Thus, only a short segment of wild-type mtDNA was retained in the mutant (Fig. $3)$. This observation is in accord with the characteristics of $S$. cerevisiae petite mtDNA where a short segment is multiplied to reach a similar size to that of 
(a)

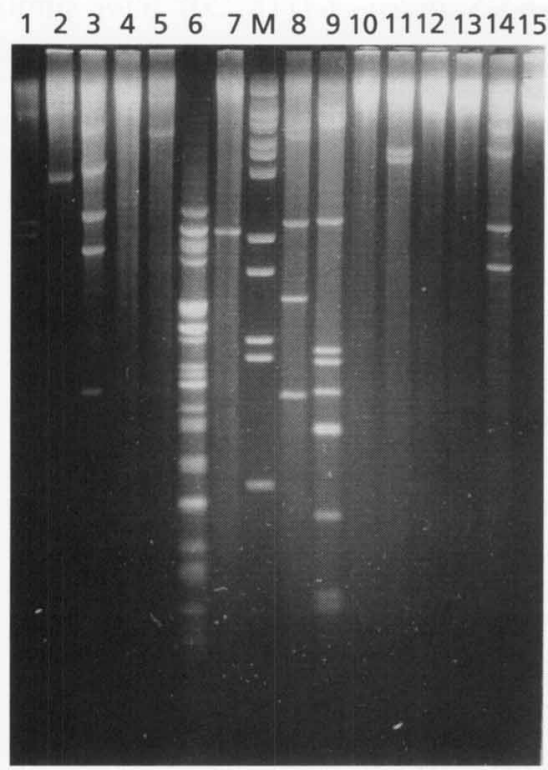

(b)

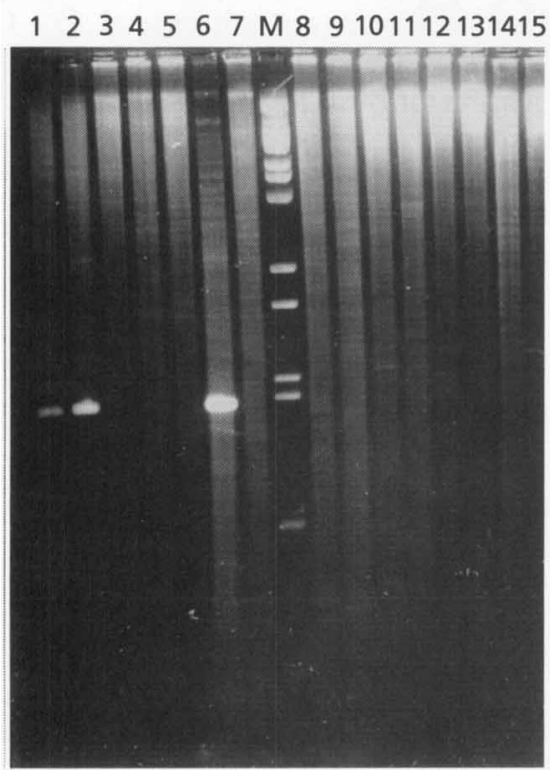

Fig. 3. mtDNA from $S$. dairensis respiration-competent wildtype strain (a) and a spontaneously generated petite strain, Y143 (b) was isolated and digested with various restriction enzymes: Accl (lane 1), BamHI (2), Bg/lI (3), Cfol (4), Clal (5), Dral (6), EcoRI (7), HindIII (8), Nsil (9), Pstl (10), Pvull (11), Sall (12), Sphl (13), Xbal (14) and Xhol (15). Fragments were separated on a $1 \%$ agarose gel. Note that petite mtDNA contains restriction sites of only three enzymes, 1, 2 and 6, which all generate a single fragment of about $1 \mathrm{~kb}$. Lambda DNA digested with BstEll was used as a size marker, $M$.

the wild-type mtDNA molecule $(2,33)$. Petite mtDNA molecules, characterized by extensive deletions, were found in all tested species from the sensu stricto as well as sensu lato groups, but not in S. kluyveri. It is of interest to note that the observed frequency of petites is not a simple function of the size and organization of the mtDNA molecules (Tables 1 and 3).

When a mitochondrial mutant is induced in $S$. cerevisiae, it takes about $8 \mathrm{~h}$ growth in a rich medium to obtain the first homoplasmic clones (14). This segregation process can be studied as induction of petites with ethidium bromide (Et-Br) $(16,33)$. When $S$. cerevisiae was treated with Et-Br, practically all colonies turned into petites after $8 \mathrm{~h}$ growth at a very low concentration of Et-Br (Table 3). Such a high inducibility was also found among all yeasts belonging to the sensu stricto group (Table 3). At higher concentrations of Et-Br almost all colonies turned into petites, and at concentrations above $50 \mathrm{mg} \mathrm{l}^{-1}$ the cells did not survive the treatment.

Inducibility of petites in the sensu lato yeasts exhibited a different pattern from that of sensu stricto yeasts (Table 3). After attempted induction with sublethal concentrations of Et-Br (50 mg $1^{-1}$ and below), a majority of cells were found to be respiration-competent. The frequency of petites was only moderately elevated, i.e. for $S$. castellii the highest frequency obtained upon induction was about fourfold higher than the spontaneous frequency (Table 3 ). By comparison, in sensu stricto yeasts the frequency of petites at equal concentrations of $\mathrm{Et}-\mathrm{Br}$ reached $100 \%$. Concentrations above $50 \mathrm{mg} \mathrm{l}^{-1}$ were lethal for sensu lato yeasts as they were for $S$. cerevisiae. When the exposure to $\mathrm{Et}-\mathrm{Br}$ was prolonged from 8 to $20 \mathrm{~h}$ at concentrations of $25-50 \mathrm{mg} \mathrm{Et}-\mathrm{Br} \mathrm{l}{ }^{-1}$, the frequency of petites increased and passed $50 \%$ in all sensu lato yeasts, except for $S$. kluyveri (data not shown).

In the case of $S$. kluyveri no petites could be induced. Even after prolonged growth at $50 \mathrm{mg} \mathrm{Et}-\mathrm{Br} \mathrm{l}^{-1}$, all surviving colonies were respiration-competent. On the basis of the above experiments (Table 3 ) the yeasts belonging to the genus Saccharomyces could be divided into three groups: (i) highly inducible yeasts of the sensu stricto group, (ii) moderately inducible yeasts of the sensu lato group, and (iii) $S$. kluyveri which is a petite-negative yeast.

\section{Origin of the modern mtDNA molecules}

The genus Saccharomyces at present contains ten recognized species $(1,20)$, but only in one of them, $S$. cerevisiae, has the nuclear genome been thoroughly characterized (for a review, see 15). Karyotypes of some other species have been partially characterized $(24,35)$, and partial sequences of nuclear rRNA genes have been determined $(18,20)$. The data on mtDNA molecules have previously been limited to some isolates of $S$. cerevisiae (for a review, see 37), and single isolates of S. douglasii (34), S. exiguus (7) and S. kluyveri (23). In the literature it is also possible to find some characterization of the yeast strain, NCYC74, which was incorrectly designated as $S$. carlsbergensis $(29,32)$. However, this strain was later suggested and shown to be an isolate of $S$. cerevisiae (19). Thus, mtDNA of $S$. 
carlsbergensis has not been characterized previously. Based on various molecular comparisions, it appears that so far the sensu stricto complex has four species: $S$. cerevisiae, $S$. paradoxus (synonym $S$. douglasii), $S$. bayanus (synonym $S$. uvarum) and a hybrid, $S$. pastorianus (synonym $S$. carlsbergensis). The other six species belong to the sensu lato group (1). However, there is still much uncertainty regarding the phylogenetic relationship among these as well as other isolates belonging to the genus Saccharomyces.

When mtDNA isolated from various Saccharomyces yeasts was digested with various restriction enzymes unique restriction patterns were observed for each isolate (Figs 1 and 2). It is interesting to stress that in $S$. cerevisiae HaeIII and MspI cut predominantly in the intergenic sequences which are more variable among various strains than the coding regions (37). When several isolates of $S$. cerevisiae were compared for their HaeIII and MspI restriction patterns, only about $50 \%$ of the bands were identical $(25,32)$. Thus, polymorphism can be expected among different isolates belonging to the same species. When different species were analysed, HaeIII and MspI generated specific finger print patterns which could be used for identification and classification of the same, as well as related isolates/species (Figs 1 and 2). Among isolates belonging to the sensu stricto group of yeasts similar restriction patterns were observed in a few cases and in general they confirmed previous molecular comparisons $(20,24)$. A similar restriction pattern was observed in $S$. carlsbergensis, $S$. monacensis and $S$. pastorianus (Figs 1 and 2), which implies that these mtDNA molecules have a common origin. $S$. carlsbergensis and $S$. pastorianus have previously been described as hybrid yeasts which were generated by an interspecific fusion/cross between two different yeasts $(19,20)$. One of the parental strains in this 'fusion/ cross' was $S$. cerevisiae, and $S$. monacensis was suggested as the second one $(17,24)$. It is interesting to point out that both sets of parental chromosomes are present in the case of nuclear DNA (19), while only the non- $S$. cerevisiae mtDNA can be found in $S$. carlsbergensis/S. pastorianus (Figs 1 and 2). This mtDNA contains a similar number of ori/rep sequences to $S$. bayanus, $S$. monacensis and $S$. uvarum (Table 2). Presumably, S. pastorianus/carlsbergensis inherited mtDNA only from the non-S. cerevisiae parent. However, only further characterization of these isolates will provide more insight into the origin of the above hybrids.

The sensu lato yeasts contain smaller mtDNA molecules which do not contain an excess of HaeIII and $M s p I$ restriction sites (Table 1). This group shows more variability regarding the size of mtDNA than the sensu stricto group. Also the restriction patterns of the sensu lato group differ widely (Figs 1 and 2). The size of the mtDNA varies from $23 \mathrm{~kb}$ in $S$. exiguus, which apparently does not contain extensive intergenic sequences, to $48 \mathrm{~kb}$ in $S$. dairensis and $49 \mathrm{~kb}$ in $S$. kluyveri (Table 1). The latter two yeasts are not closely related $(18,20)$. Two more closely related species, $S$. servazzii and $S$. unisporus $(18,20)$, have similarly sized mtDNA $(29 \mathrm{~kb})$. On the other hand, another pair of closely related species, $S$. castellii and $S$. dairensis (18, 20 ), show a twofold difference in size. In general, it seems that the size of mtDNA is very variable within the genus and could have changed frequently during evolution of each modern sensu lato species. The mechanism determining the size of mtDNA is not known at present. The difference between the predicted and observed number of HaeIII and MspI sites in sensu lato species is not substantial (about two and a half times), which is very different from the situation in the sensu stricto group (Tables 1 and 2). The sensu lato group also did not give any signal with the ori/rep probe, suggesting that this sequence is not present in these yeasts. Therefore, it is expected that intergenic sequences in sensu lato species consist predominantly of A+T-rich stretches. Apparently, the type and abundance of G + C-clusters which carry HaeIII and/ or $M s p$ I sites, and especially ori/rep, evolved in the mtDNA molecule after separation of the sensu stricto and sensu lato yeasts. At this point it is interesting to mention that different mitochondrial $\mathrm{G}+\mathrm{C}$ clusters evolved several times among Ascomycetes. For example, Kluyveromyces lactis, a fairly close relative of the genus Saccharomyces, contains a different type of $\mathrm{G}+\mathrm{C}$ cluster which is recognized by $\operatorname{SacII}(30)$. While in general it is still unclear what kind of role different $\mathrm{G}+\mathrm{C}$ clusters play in the genome, it seems that in $S$. cerevisiae some of the $\mathrm{G}+\mathrm{C}$ clusters, especially ori/rep, are actively involved in transmission of $\mathrm{mtDNA}$ to progeny $(4,25,40)$. In this way certain $\mathrm{G}+\mathrm{C}$ clusters may be actively preserved in mitochondrial genomes.

However, although fewer $\mathrm{G}+\mathrm{C}$ clusters are present in the sensu lato group, this does not stabilize their mtDNA against spontaneous generation of petite mtDNA molecules (Fig. 3). Spontaneous respirationdeficient mutants were found at high frequency in all isolates, except $S$. kluyveri (Table 3, Fig. 3). This yeast behaves as a petite-negative even upon strong induction with Et-Br. This characteristic, which distinguishes $S$. kluyveri from the rest of the Saccharomyces yeasts, is supported by other observations $(20,35)$ which also suggest that this yeast should belong to a separate genus. Some other genera, i.e. Kluyveromyces, Zygosaccharomyces and Torulaspora, which are close relatives of Saccharomyces, also seem to be petitenegative (data not shown). Thus, it seems that the petite-positive character evolved specifically in the progenitor of the sensu lato and sensu stricto yeasts after separation from $S$. kluyveri and other related genera.

In conclusion, the following evolutionary development of the mitochondrial genome in the Saccharomyces yeasts is proposed. The mtDNA molecule in the progenitor of the Saccharomyces yeasts was relatively small and genetically stable, and this yeast was petitenegative. The petite-positive character evolved after the sensu lato and sensu stricto yeasts had separated 
from $S$. kluyveri. Later, the origin of the sensu stricto group and its separation from the sensu lato group coincided with the appearance of various mitochondrial G + C-rich clusters, including ori/rep. These biologically active sequences have conributed markedly to the further evolution of the size and genetic instability of the sensu stricto mitochondrial genome.

\section{ACKNOWLEDGEMENTS}

This work was supported by the Danish Research Council and the Carlsberg Foundation. The authors thank A. Kahn and T. Nilsson-Tillgren for their stimulating comments on this work. C. Kurtzman is acknowledged for providing some of the yeast strains employed in this study.

\section{REFERENCES}

1. Barnett, J. A. (1992). The taxonomy of the genus Saccharomyces Meyen ex Reess: a short review for nontaxonomists. Yeast 8, 1-23.

2. Bernardi, G. (1979). The petite mutation in yeast. Trends Biochem Sci 4, 197-201.

3. Bingham, C. G. \& Nagley, P. (1983). A petite mitochondrial DNA segment arising in exceptional high frequency in a mit-mutant of Saccharomyces cerevisiae. Biochim Biophys Acta 740, 88-98.

4. Blanc, H. \& Dujon, B. (1980). Replicator regions of the yeast mitochondrial DNA responsible for suppressiveness. Proc Natl Acad Sci USA 77, 3942-3946.

5. Clark-Walker, G. D. (1985). Basis of diversity in mitochondrial DNAs. In The Evolution of Genome Size, pp. 277-297. Edited by T. Cavalier-Smith. London: Wiley.

6. Clark-Walker, G. D., McArthur, C. R. \& Daley, D. J. (1981). Does mitochondrial DNA length influence the frequency of spontaneous petite mutants in yeasts? Curr Genet 4, 7-12.

7. Clark-Walker, G. D., McArthur, C. R. \& Sriprakash, K. S. (1983). Order and orientation of genic sequences in circular mitochondrial DNA from Saccharomyces exiguus: implications for evolution of yeast mtDNAs. J Mol Evol 19, 333-341.

8. Dieckmann, C. L. \& Gandy, B. (1987). Preferential recombination between GC clusters in yeast mitochondrial DNA. EMBO J 6, 4197-4203.

9. Dujon, B. (1981). Mitochondrial genetics and functions. In The Molecular Biology of the Yeast Saccharomyces: Life Cycle and Inheritance, pp. 505-635. Edited by J. N. Strathern, E. W. Jones \& J. R. Broach. Cold Spring Harbor, NY : Cold Spring Harbor Laboratory.

10. Evans, R. J., Oakley, K. M. \& Clark-Walker, G. D. (1985). Elevated levels of petite formation in strains of Saccharomyces cerevisiae restored to respiratory competence. I. Association of both high and moderate frequencies of petite mutant formation with the presence of aberrant mitochondrial DNA. Genetics 111, 389-402.

11. Fangman, W. L., Henly, J. W., Churchill, G. \& Brewer, B. J. (1989). Stable maintenance of a 35-base-pair yeast mitochondrial genome. Mol Cell Biol 9, 1917-1921.

12. Faugeron-Fonty, G. \& Goyon, C. (1985). Polymorphic variations in the ori sequences from the mitochondrial genomes of different wild-type yeast strains. Curr Genet 10, 269-282.
13. Ferguson, L. R. \& von Borstel, R. C. (1992). Induction of the cytoplasmic 'petite' mutation by chemical and physical agents in Saccharomyces cerevisiae. Mutat Res 265 , 103-148.

14. Gingold, E. B. (1988). The replication and segregation of yeast mitochondrial DNA. In Division and Segregation of Organelles (Society for Experimental Biology Seminar Series, vol. 35), pp. 149-170. Edited by S. A. Boffey \& D. Lloyd. Cambridge: Cambridge University Press.

15. Goffeau, A., Barrell, B. G., Bussey, H. \& 13 other authors (1996). Life with 6000 genes. Science 274, 546-567.

16. Hall, R. M., Trembath, M. K., Linnane, A. W., Wheelis, L. \& Criddle, R. S. (1976). Factors affecting petite induction and the recovery of respiratory competence in yeast cells exposed to ethidium bromide. Mol Gen Genet 144, 253-262.

17. Hansen, J. \& Kielland-Brandt, M. C. (1994). Saccharomyces carlsbergensis contains two functional $M E T 2$ alleles similar to homologues from $S$. cerevisiae and $S$. monacensis. Gene 140, 33-40.

18. James, S. A., Cai, J., Roberts, I. N. \& Collins, M. D. (1997). A phylogenetic analysis of the genus Saccharomyces based on 18S rRNA gene sequences: description of Saccharomyces kunashirensis sp. nov. and Saccharomyces martiniae sp. nov. Int J Syst Bacteriol 47, 453-460.

19. Kielland-Brandt, M., Nilsson-Tillgren, T., Gjermansen, C., Holmberg, S. \& Pedersen, M. B. (1995). Genetics of brewing yeasts. In The Yeasts, vol. 6, pp. 223-254. Edited by A. H. Rose, A. E. Wheals \& J. S. Harrison. London: Academic Press.

20. Kurtzman, C. P. \& Robnett, C. J. (1991). Phylogenetic relationship among species of Saccharomyces, Schizosaccharomyces, Debaryomyces and Schwanniomyces determined from partial ribosomal RNA sequences. Yeast 7 , 61-72.

21. Mandel, M., Schildkraut, C. L. \& Marmur, L. (1968). Use of $\mathrm{CsCl}$ density gradient analysis for determining the guanine plus cytosine content of DNA. Methods Enzymol 12B, 184-195.

22. Ogur, M., John, S.T. R. \& Nagai, S. (1957). Tetrazolium overlay technique for population studies of respiration deficiency in yeast. Science 125, 928-929.

23. Okamoto, K., Suzuki, K. \& Yoshida, K. (1991). Physical mapping and RFLP analysis of mtDNAs from the ascosporogenous yeast: Saccharomyces exiguus, $S$. kluyveri and Hansenula wingei. Jpn J Genet 66, 709-718.

24. Pedersen, M. B. (1986). DNA sequence polymorphism in the genus Saccharomyces. IV. Homologous chromosomes III in Saccharomyces bayanus, S. carlsbergensis, and S. uvarum. Carlsberg Res Commun 51, 185-202.

25. Piצ̌kur, J. (1988). Transmission of yeast mitochondrial loci to progeny is reduced when nearby intergenic regions containing ori/rep sequences are deleted. Mol Gen Genet 214, 425-432.

26. Piłkur, J. (1989a). Transmission of the yeast mitochondrial genome to progeny: the impact of intergenic sequences. Mol Gen Genet 218, 161-168.

27. Piצkkur, J. (1989b). Respiratory-competent yeast mitochondrial DNAs generated by deleting intergenic region. Gene 81, 165-168.

28. Piškur, J. (1994). Inheritance of the yeast mitochondrial genome. Plasmid 31, 229-241.

29. Prunell, A., Kopecka, H., Strauss, F. \& Bernardi, G. (1977). The 
mitochondrial genome of wild-type yeast cells. V. Genome evolution. J Mol Biol 110, 17-52.

30. Ragnini, A. \& Fukuhara, H. (1988). Mitochondrial DNA of the yeast Kluyveromyces: guanine-cytosine rich sequence clusters. Nucleic Acids Res 16, 8433-8442.

31. Rayko, E., Goursot, R., Cherif-Zahar, B., Melis, R. \& Bernardi, G. (1988). Regions flanking ori sequences affect replication efficiency of the mitochondrial genome of ori-plus petite mutant from yeast. Gene 63, 213-226.

32. Sanders, J. P. M., Heyting, C., Verbeet, M. Ph., Meijlink, F. C. P. W. \& Borst, P. (1977). The organization of genes in yeast mitochondrial DNA. III. Comparison of the physical maps of the mitochondrial DNAs from three wild-type Saccharomyces strains. Mol Gen Genet 157, 239-261.

33. Sor, F. \& Fukuhara, H. (1983). Unequal excision of complementary strands is involved in the generation of palindromic repetitions of rho $^{-}$mitochondrial DNA in yeast. Cell 32, 391-396.

34. Tian, G.-L., Macadre, C., Kruszewska, A. \& 7 other authors (1991). Incipient mitochondrial evolution in yeasts. I. The physical map and gene order of Saccharomyces douglasii mitochondrial DNA discloses a translocation of a segment of 15000 base-pairs and the presence of new introns in comparison with Saccharomyces cerevisiae. J Mol Biol 218 , 735-746.

35. Vaughan-Martini, A., Martini, A. \& Cardinali, G. (1993). Electrophoretic karyotyping as a taxonomic tool in the genus Saccharomyces. Antonie Leeuwenhoek 63, 145-156.

36. Wolf, K. \& Del Guidice, L. (1988). The variable mitochondrial genome of Ascomycetes: organization, mutational alterations, and expression. Adv Genet 25, 185-308.

37. de Zamaroczy, M. \& Bernardi, G. (1985). Sequence organization of the mitochondrial genome of yeast - a review. Gene 37, 1-17.

38. de Zamaroczy, M. \& Bernardi, G. (1986). The GC clusters of the mitochondrial genome of yeast and their evolutionary origin. Gene 41, 1-22.

39. de Zamaroczy, M., Faugeron-Fonty, G. \& Bernardi, G. (1983). Excision sequences in the mitochondrial genome of yeast. Gene 21, 193-202.

40. de Zamaroczy, M., Marotta, R., Faugeron-Fonty, G., Goursot, R., Mangin, M., Baldacci, G. \& Bernardi, G. (1981). The origins of replication of the yeast mitochondrial genome and the phenomenon of suppressivity. Nature 292, 75-78. 